\title{
Effectiveness and Safety of Pegfilgrastim in BEP Treatment for Patients with Germ Cell Tumor
}

\author{
HIROAKI IWAMOTO, KOUJI IZUMI, ARIUNBOLD NATSAGDORJ, TOMOYUKI MAKINO, \\ TAKAHIRO NOHARA, KAZUYOSHI SHIGEHARA, YOSHIFUMI KADONO and ATSUSHI MIZOKAMI \\ Department of Integrative Cancer Therapy and Urology, \\ Kanazawa University Graduate School of Medical Science, Kanazawa, Japan
}

\begin{abstract}
Background: The effectiveness and safety of pegfilgrastim during bleomycin, etoposide and cisplatin (BEP) chemotherapy have not yet been investigated. Patients and Methods: Patients with germ cell tumors (GCTs) who received pegfilgrastim during $B E P$ at the Kanazawa University Hospital between January 2014 and December 2016 were retrospectively analyzed. The frequency of adverse events and effectiveness in inhibiting neutropenia were compared between cycles using pegfilgrastim and those using filgrastim. Results: Pegfilgrastim and filgrastim were administered in 13 and 22 cycles, respectively. The absolute neutrophil count at the nadir was significantly lower in patients receiving pegfilgrastim than in those receiving filgrastim ( $p=0.003)$. The duration of grade 2-4 neutropenia in cycles using filgrastim was significantly longer than that in those pegfilgrastim $(p=0.01)$. No significant differences in the incidence of febrile neutropenia and serious adverse events were observed. Conclusion: Pegfilgrastim can be safely and effectively administrated during BEP for patients with GCT.
\end{abstract}

Germ cell tumor (GCT) is a common malignancy that affects adolescent and young adult males. The development of an effective combined chemotherapy, specifically bleomycin, etoposide and cisplatin (BEP), has dramatically improved the prognosis of patients with advanced GCT $(1,2)$. However, BEP induces severe myelosuppression. When administering

This article is freely accessible online.

Correspondence to: Kouji Izumi, MD, Ph.D., Department of Integrative Cancer Therapy and Urology, Kanazawa University Graduate School of Medical Science, Kanazawa, 13-1 Takara-machi Kanazawa, Ishikawa, 920-8640 Japan. Tel: +81 762652393, Fax: +81 762226726, Email: azuizu2003@yahoo.co.jp

Key Words: BEP, germ cell tumor, neutropenia, pegfilgrastim. the standard BEP regimen without granulocyte-colony stimulating factor (G-CSF), 30-50\% of treated patients are generally unable to receive full-dose treatment as scheduled because of myelosuppression (3). Because worse outcomes are reported with insufficient BEP dose intensity, G-CSF, such as filgrastim, is administered daily during BEP in most cases (4). Filgrastim allows the timely delivery of cytotoxic chemotherapy at an adequate dose (5-7). Pegfilgrastim, a sustained-release form of filgrastim, reduces the number of injections required to one per cycle of chemotherapy. Many studies have demonstrated the noninferiority of pegfilgrastim compared to filgrastim in terms of the duration of severe neutropenia and risk of febrile neutropenia $(\mathrm{FN})(8,9)$. However, to our knowledge, no study has reported on the use of pegfilgrastim during the standard BEP treatment for GCT. In this study, the effectiveness and safety of pegfilgrastim compared to filgrastim during BEP for GCT were investigated.

\section{Patients and Methods}

Between January 2014 and December 2016, 16 patients with GCT were treated at Kanazawa University Hospital. Six patients who had not received BEP were excluded from the study. Consequently, 10 patients met the inclusion criterion and were retrospectively analyzed. The demographic, surgical, pathological, and follow-up data were collected from their medical charts. Patients received a BEP regimen, composed of $30 \mathrm{mg}(30,000$ international units $)$ intravenous bleomycin (i.v.) injection on days 1,8 , and $15 ; 100 \mathrm{mg} / \mathrm{m}^{2}$ etoposide i.v. on days $1,2,3,4$, and 5 ; and $20 \mathrm{mg} / \mathrm{m}^{2}$ cisplatin i.v. on days 1 , $2,3,4$, and 5 . Treatment was repeated every 3 weeks for two to four cycles. Pegfilgrastim $(3.6 \mathrm{mg}$ ) was administered subcutaneously on day 7 . The administration of filgrastim $(75 \mu \mathrm{g})$ was at the discretion of each attending physician. Leukopenia, neutropenia, thrombocytopenia, anemia, FN, and other adverse events were analyzed. Statistical analyses were performed using commercially available software Prism (GraphPad, San Diego, CA, USA). Comparisons among different groups were performed using the chisquared test and the Mann-Whitney $U$-test. In all analyses, $p$-values of less than 0.05 indicated statistical significance. 
Table I. Patient background characteristics.

\begin{tabular}{|c|c|c|c|c|c|c|}
\hline \multirow[t]{2}{*}{ No. } & \multirow[t]{2}{*}{ Age, years } & \multirow[t]{2}{*}{ Pathology } & \multirow[t]{2}{*}{ IGCC } & \multirow[t]{2}{*}{ Distant metastasis } & \multicolumn{2}{|c|}{ No. of cycles } \\
\hline & & & & & Peg & Filg \\
\hline 1 & 28 & Non-seminomatous & Poor & LN, lung & 3 & 1 \\
\hline 2 & 47 & Mixed & Good & None & 1 & 1 \\
\hline 3 & 56 & Mixed & Intermediate & None & $2 *$ & 2 \\
\hline 4 & 48 & Mixed & Good & None & 4 & 0 \\
\hline 5 & 30 & Seminoma & Good & None & 3 & 0 \\
\hline 6 & 48 & Seminoma & Good & None & 0 & 3 \\
\hline 7 & 19 & Non-seminomatous & Poor & LN, lung & 0 & 4 \\
\hline 8 & 44 & Seminoma & Good & None & 0 & 3 \\
\hline 9 & 32 & Non-seminomatous & Poor & LN & 0 & 4 \\
\hline 10 & 37 & Mixed & Poor & LN, lung & 0 & 4 \\
\hline
\end{tabular}

*Filgrastim was administered following pegfilgrastim in one cycle. Mixed: Non-seminomatous and seminoma; IGCC: International Germ Cell Classification; BEP: bleomycin + etoposide + cisplatin; Peg: pegfilgrastim; Filg: filgrastim; LN: lymph node.

Table II. Hematological adverse events of therapy with bleomycin, etoposide and cisplatin, according to administration of granulocyte-colony stimulating factor.

\begin{tabular}{|c|c|c|c|}
\hline & Pegfilgrastim $(3.6 \mathrm{mg})$ & Filgrastim $(75 \mu \mathrm{g})$ & $p$-Value \\
\hline Cycles administered, $\mathrm{n}$ & 13 & 22 & \\
\hline Median no. of administrations per cycle & 1 & $5(1-9)$ & \\
\hline Median baseline ANC $\left(10^{3} / \mu \mathrm{l}\right)$ & $6.31(2.07-8.99)$ & $5.73(1.58-24.2)$ & 0.52 \\
\hline ANC at nadir $\left(10^{3} / \mu \mathrm{l}\right)$ & $1.99(0.4-5.1)$ & $0.82(0.06-2.1)$ & 0.003 \\
\hline \multicolumn{4}{|l|}{ Grade 4 adverse event } \\
\hline Anemia & 0 & $1(4.5 \%)$ & $>0.99$ \\
\hline Thrombocytopenia & 0 & 0 & \\
\hline Leukopenia & 0 & $5(22.7 \%)$ & 0.13 \\
\hline Neutropenia & $1(14.3 \%)$ & $8(36.4 \%)$ & 0.11 \\
\hline \multicolumn{4}{|l|}{ Grade 3 or more } \\
\hline Febrile neutropenia & 0 & $2(9.1 \%)$ & 0.51 \\
\hline
\end{tabular}

ANC: Absolute neutrophil count.

\section{Results}

Of 10 patients who met the inclusion criterion, pegfilgrastim and filgrastim were administered to five and eight patients, respectively. Three patients received both pegfilgrastim and filgrastim during BEP treatment. One patient received both pegfilgrastim and filgrastim in the same cycle. In total, pegfilgrastim and filgrastim were administered in 13 and 22 cycles, respectively. The median age at GCT diagnosis was 40.5 years. Three patients were found to have seminoma, four had mixed tumors of seminoma and nonseminoma, and three had nonseminoma. According to International Germ Cell Classification (10), five, one, and four patients were classified with good, intermediate, and poor prognosis, respectively (Table I).
Hematologic adverse events are shown in Table II. There was no significant difference in baseline absolute neutrophil count (ANC) between cycles using pegfilgrastim and those using filgrastim $(p=0.52)$; nevertheless, the nadir ANC value was significantly lower in those using filgrastim $(p=0.003)$. The line graphs of the number of neutrophils in all cycles using both agents are shown in Figure 1. Although there was no significant difference, FN occurred in two cycles using filgrastim and did not occur in any cycles using pegfilgrastim. The duration of grade 2-4 neutropenia in cycles using filgrastim was significantly longer than in those using pegfilgrastim ( $p=0.01$, median of 0 and 2 days, respectively, Figure 2). Serious adverse events, such as interstitial pneumonia and splenic rupture, were not observed. However, more severe neutrophilia was observed in cycles using 


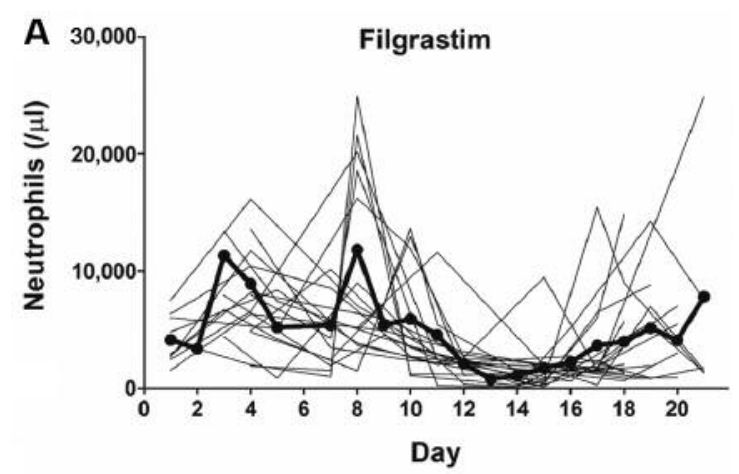

B

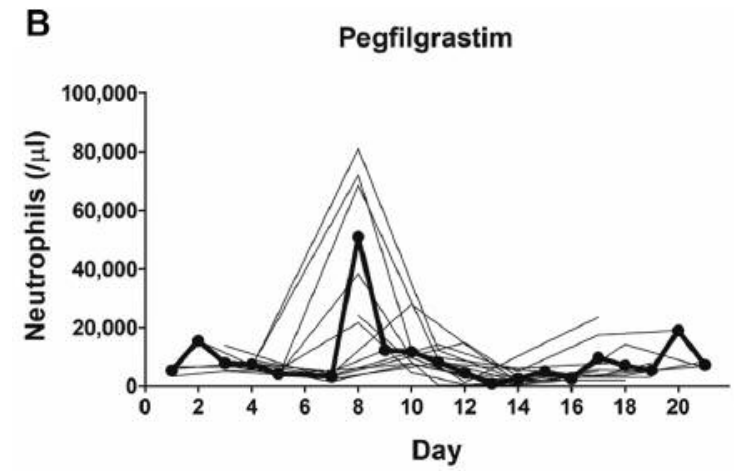

Figure 1. The number of neutrophils in all cycles in individual patients treated with bleomycin, etoposide and cisplatin, according to administration of pegfilgrastim (A) and filgrastim (B), respectively. Bold lines indicate the average values each day.

pegfilgrastim than in those using filgrastim (median 21,800 and $13,500 / \mu \mathrm{l}$, respectively, $p=0.006$; Figure $3 \mathrm{~A}$ ). The median number of days to maximum neutrophil count (MNC) in cycles using pegfilgrastim and those using filgrastim was 8 and six, respectively (Figure 3B). Although there was no significant difference, MNC most frequently occurred after pegfilgrastim injection (11 out of $13,85 \%$ ). MNC was recorded before the initiation of filgrastim injection in 50\% (11 out of 22) of filgrastim-used cycles (Figure 3C). Advanced GCT is sometimes associated with systemic inflammation with high baseline ANC before the induction of BEP treatment. The cycle which included the day of MNC before the initiation of pegfilgrastim and filgrastim was recorded for each patient. The percentage of patients with MNC before the initiation of filgrastim injection in the first cycle and subsequent cycles was $27 \%$ and $73 \%$, respectively (not significantly different, Figure 3D).

\section{Discussion}

Pegfilgrastim is generally administered between 14 days before and $24 \mathrm{~h}$ after the administration of chemotherapy agents. In this study, patients receive pegfilgrastim on day 7

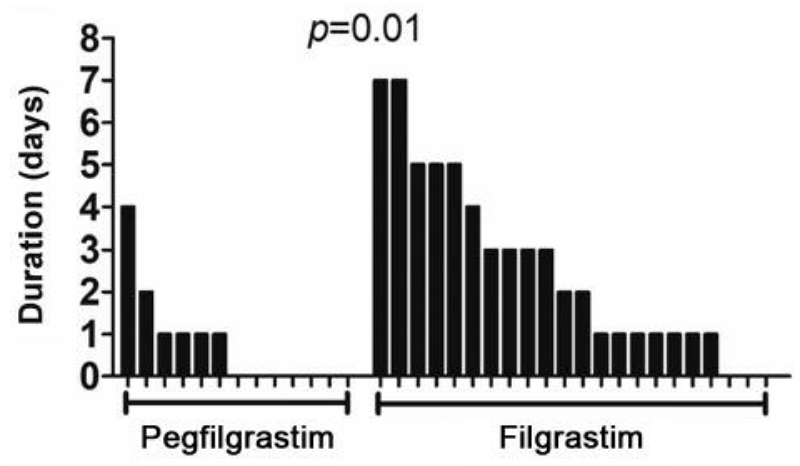

Figure 2. The duration of grade 2-4 neutropenia in individual patients treated with bleomycin, etoposide and cisplatin, according to administration of granulocyte-colony stimulating factor.

in the BEP regimen, that is, 2 days after etoposide and cisplatin administration. This shows the effect of pegfilgrastim during days 8 and 15 when bleomycin is administered, because pegfilgrastim is a sustained-release form of filgrastim. Theoretically, simultaneous administration of exogenous G-CSF and chemotherapy leads to an increased pool of neutrophil precursors susceptible to destruction by chemotherapy, which paradoxically leads to an increased risk of neutropenia $(11,12)$. In a retrospective study, Weycker $e t$ $a l$. analyzed 45,592 patients who received pegfilgrastim. They reported that FN incidence was found to be significantly higher in patients who received pegfilgrastim prophylaxis on the same day as chemotherapy completion compared to those who received it several days after the completion of chemotherapy (13). In contrast, Burris et al. reviewed three randomized double-blind studies comparing same-day and next-day pegfilgrastim and showed a statistically insignificant trend toward longer duration of severe neutropenia for the same-day group (14). Bleomycin was reported to lead to minimal or no myelosuppression and leukopenia in vitro, and neutropenia rarely occurred when it was used as a singleagent treatment $(15,16)$. The simultaneous use of bleomycin and G-CFS was reported to increase the risk of interstitial pneumonia in patients with lymphoma; however, an increase in bleomycin-induced pulmonary toxicity has not been reported when using G-CSF in GCT chemotherapy regimens containing bleomycin $(3,17,18)$. These results may support the use of pegfilgrastim during BEP treatment, particularly when bleomycin is administered under the lasting effect of sustained-release pegfilgrastim.

Interestingly, the MNC was recorded most frequently after pegfilgrastim injection in $85 \%$ (11 out of 13) of cycles using pegfilgrastim. In contrast, it was recorded before the initiation of filgrastim injection in 50\% (11 out of 22) of cycles using filgrastim. These results indicate that filgrastim injection may 
A

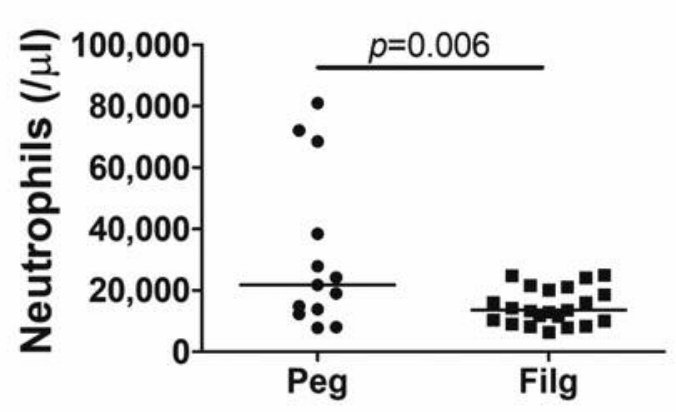

C

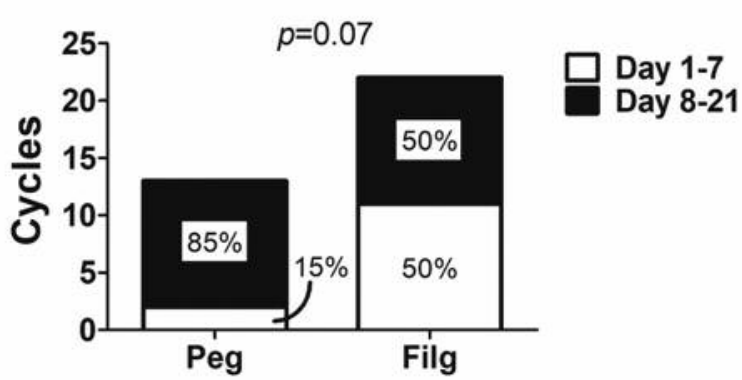

B

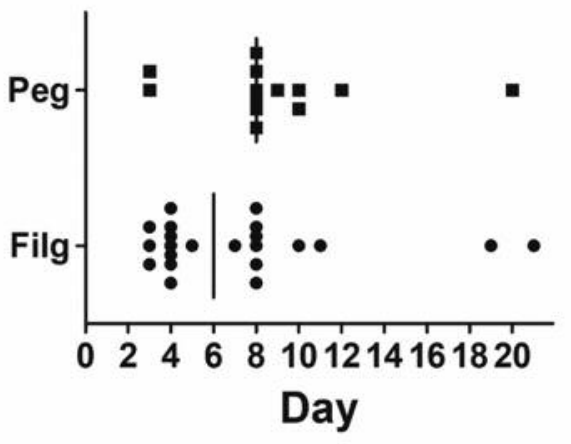

D

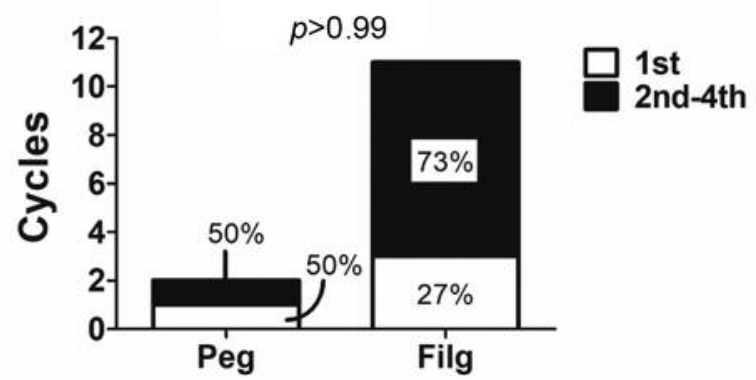

Figure 3. Changes in neutrophil count in patients treated with bleomycin, etoposide and cisplatin during cycles using pegfilgrastim (Peg) and filgrastim (Fil). A: Maximum neutrophil count for individual patients. Bars represent median values. B: The number of days to the maximum neutrophil count for individual patients. C: The percentage of patients with maximum neutrophil count before and after initiation of granulocytecolony stimulating factor $(G-C S F)$ is shown. Most maximum neutrophil counts were recorded on days after pegfilgrastim injection in cycles administering pegfilgrastim; however, only half of the maximum neutrophil counts were recorded after filgrastim injection in cycles administering filgrastim. D: Of cycles with maximum neutrophil count recorded before initiation of G-CSF, the percentage of cycles with maximum count before G-CSF initiation in the first and subsequent (second, third, and fourth) cycles in the therapy series of bleomycin, etoposide, and cisplatin is shown.

not be efficacious for increasing the neutrophil count in patients under BEP treatment. Furthermore, the proportion of the median number of days to MNC before the initiation of filgrastim injection in the first cycle is only $27 \%$, indicating that $\mathrm{MNC}$ early in the filgrastim cycle is not due to the systemic inflammation caused by active GCT before BEP treatment, that is, filgrastim may not be able to increase the neutrophil count sufficiently beyond the baseline number of neutrophils before the initiation of filgrastim injection. In contrast, even in pegfilgrastim-induced neutrophilia, the number of leukocytes was less than $100,000 / \mu \mathrm{l}$, which is borderline grade 3 neutrophilia. Moreover, neither interstitial pneumonia nor splenic rupture were observed. Splenic rupture secondary to GCSF use most commonly occurs in patients and healthy donors in the hematopoietic cell transplantation setting (19-22).

This study had several limitations. The median number of administrations of filgrastim was five, while administration of pegfilgrastim once per cycle is equivalent to a daily injection of filgrastim for 11 days (9). This difference potentially leads to differences between cycles using pegfilgrastim and those using filgrastim. The small sample size may have prevented determination of the precise statistical significance of differences between cycles using the two different forms of G-CSF. Larger prospective studies with longer follow-up periods and data from other ethnic backgrounds are needed to confirm the findings of this study. In conclusion, this study showed that pegfilgrastim during BEP for patients with GCT may be a potential treatment to effectively reduce the severity and duration of neutropenia with minimal toxicity.

\section{Conflicts of Interest}

The Authors declare that they have no conflicts of interest.

\section{References}

1 Williams SD, Birch R, Einhorn LH, Irwin L, Greco FA and Loehrer PJ: Treatment of disseminated germ-cell tumors with 
cisplatin, bleomycin, and either vinblastine or etoposide. N Engl J Med 316: 1435-1440, 1987.

2 Shamash J, Sarker SJ, Huddart R, Harland S, Joffe JK, Mazhar D, Birtle A, White J, Chowdhury K, Wilson P, Marshall MR and Vinnicombe S: A randomized phase III study of $72 \mathrm{~h}$ infusional versus bolus bleomycin in BEP (bleomycin, etoposide and cisplatin) chemotherapy to treat IGCCCG good prognosis metastatic germ cell tumours (TE-3). Ann Oncol 28: 1333-1338, 2017.

3 Fossa SD, Kaye SB, Mead GM, Cullen M, de Wit R, Bodrogi I, van Groeningen CJ, De Mulder PH, Stenning S, Lallemand E, De Prijck L and Collette L: Filgrastim during combination chemotherapy of patients with poor-prognosis metastatic germ cell malignancy. European Organization for Research and Treatment of Cancer, Genito-Urinary Group, and the Medical Research Council Testicular Cancer Working Party, Cambridge, United Kingdom. J Clin Oncol 16: 716-724, 1998.

4 Toner GC, Stockler MR, Boyer MJ, Jones M, Thomson DB, Harvey VJ, Olver IN, Dhillon H, McMullen A, Gebski VJ, Levi JA and Simes RJ: Comparison of two standard chemotherapy regimens for good-prognosis germ-cell tumours: a randomised trial. Australian and New Zealand Germ Cell Trial Group. Lancet 357: 739-745, 2001.

5 Trillet-Lenoir V and Green J, Manegold C, Von Pawel J, Gatzemeier U, Lebeau B, Depierre A, Johnson P, Decoster G, Tomita D and Ewen C: Recombinant granulocyte colonystimulating factor reduces the infectious complications of cytotoxic chemotherapy. Eur J Cancer 29A: 319-324, 1993.

6 Pettengell R, Gurney H, Radford JA, Deakin DP, James R, Wilkinson PM, Kane K, Bentley J and Crowther D: Granulocyte colony-stimulating factor to prevent dose-limiting neutropenia in non-Hodgkin's lymphoma: a randomized controlled trial. Blood 80: 1430-1436, 1992.

7 Crawford J, Ozer H, Stoller R, Johnson D, Lyman G, Tabbara I, Kris M, Grous J, Picozzi V, Rausch G, Smith R, Gradishar W, Yahanda A, MVincent M, Stewart M and Glaspy J: Reduction by granulocyte colony-stimulating factor of fever and neutropenia induced by chemotherapy in patients with small-cell lung cancer. N Engl J Med 325: 164-170, 1991.

8 Green MD, Koelbl H, Baselga J, Galid A, Guillem V, Gascon P, Siena S, Lalisang RI, Samonigg H, Clemens MR, Zani V, Liang BC, Renwick J, Piccart MJ and International Pegfilgrastim 749 Study Group: A randomized double-blind multicenter phase III study of fixed-dose single-administration pegfilgrastim versus daily filgrastim in patients receiving myelosuppressive chemotherapy. Ann Oncol 14: 29-35, 2003.

9 Kubo K, Miyazaki Y, Murayama T, Shimazaki R, Usui N, Urabe A, Hotta T and Tamura K: A randomized, double-blind trial of pegfilgrastim versus filgrastim for the management of neutropenia during CHASE(R) chemotherapy for malignant lymphoma. Br J Haematol 174: 563-570, 2016.

10 International Germ Cell Consensus Classification: a prognostic factor-based staging system for metastatic germ cell cancers. International Germ Cell Cancer Collaborative Group. J Clin Oncol 15: 594-603, 1997.

11 Meropol NJ, Miller LL, Korn EL, Braitman LE, MacDermott ML and Schuchter LM: Severe myelosuppression resulting from concurrent administration of granulocyte colony-stimulating factor and cytotoxic chemotherapy. J Natl Cancer Inst 84: 12011203, 1992.
12 Rowinsky EK, Grochow LB, Sartorius SE, Bowling MK, Kaufmann SH, Peereboom D and Donehower RC: Phase I and pharmacologic study of high doses of the topoisomerase I inhibitor topotecan with granulocyte colony-stimulating factor in patients with solid tumors. J Clin Oncol 14: 1224-1235, 1996.

13 Weycker D, Li X, Figueredo J, Barron R, Tzivelekis S and Hagiwara M: Risk of chemotherapy-induced febrile neutropenia in cancer patients receiving pegfilgrastim prophylaxis: Does timing of administration matter? Support Care Cancer 24: 23092316, 2016.

14 Burris HA, Belani CP, Kaufman PA, Gordon AN, Schwartzberg LS, Paroly WS, Shahin S, Dreiling L and Saven A: Pegfilgrastim on the same day versus next day of chemotherapy in patients with breast cancer, non-small-cell lung cancer, ovarian cancer, and non-Hodgkin's lymphoma: results of four multicenter, double-blind, randomized phase II Studies. J Oncol Pract 6: 133140, 2010.

15 Tisman G, Herbert V, Go LT and Brenner L: Marked immunosuppression with minimal myelosuppression by bleomycin in vitro. Blood 41: 721-726, 1973.

16 Halnan KE, Bleehen NM, Brewin TB, Deeley TJ, Harrison DF, Howland C, Kunkler PB, Ritchie GL, Wiltshaw E and Todd ID: Early clinical experience with bleomycin in the United Kingdom in series of 105 patients. Br Med J 4: 635-638, 1972.

17 Kwan EM, Beck S, Amir E, Jewett MA, Sturgeon JF, AnsonCartwright L and Chung PW, Warde PR, Moore MJ, Bedard PL and Tran B: Impact of granulocyte-colony stimulating factor on bleomycin-induced pneumonitis in chemotherapy-treated germ cell tumors. Clin Genitourin Cancer, 2017. doi: 10.1016/j.clgc.2017.08.012. [Epub ahead of print]

18 Maruyama Y, Sadahira T, Mitsui Y, Araki M, Wada K, Tanimoto R, Kobayashi Y, Watanabe M, Watanabe T and Nasu Y: Prognostic impact of bleomycin pulmonary toxicity on the outcomes of patients with germ cell tumors. Med Oncol 35: 80, 2018.

19 Tigue CC, McKoy JM, Evens AM, Trifilio SM, Tallman MS and Bennett CL: Granulocyte-colony stimulating factor administration to healthy individuals and persons with chronic neutropenia or cancer: an overview of safety considerations from the Research on Adverse Drug Events and Reports project. Bone Marrow Transplant 40: 185-192, 2007.

20 Falzetti F, Aversa F, Minelli O and Tabilio A: Spontaneous rupture of spleen during peripheral blood stem-cell mobilisation in a healthy donor. Lancet 353: 555, 1999.

21 Oran B, Wright DG, Seldin DC, McAneny D, Skinner M and Sanchorawala V: Spontaneous rupture of the spleen in AL amyloidosis. Am J Hematol 74: 131-135, 2003.

22 Zimmer BM, Berdel WE, Ludwig WD, Notter M, Reufi B and Thiel E: Fatal spleen rupture during induction chemotherapy with rhGM-CSF priming for acute monocytic leukemia. Clinical case report and in vitro studies. Leuk Res 17: 277-283, 1993.

Received April 2, 2018

Revised May 2, 2018

Accepted May 3, 2018 